\title{
Hadronic thermal model with distinct freeze-out temperatures for baryons and mesons
}

\author{
Sérgio B. Duarte ${ }^{* \dagger}$ \\ Centro Brasileiro de Pesquisas Físicas \\ Rua Dr. Xavier Sigaud 150, 22290-180 Rio de Janeiro-RJ, Brasil \\ E-mail: sbdecbpf.br
}

\section{Leonardo P. G. De Assis}

Centro Brasileiro de Pesquisas Físicas

Rua Dr. Xavier Sigaud 150, 22290-180 Rio de Janeiro-RJ, Brasil

E-mail: lpgassisecbpf.br

\section{Marcelo Chiapparini}

Instituto de Física, Universidade do Estado do Rio de Janeiro Rua São Francisco Xavier 524, 20550-900 Rio de Janeiro-RJ, Brasil

E-mail: chiappa@uerj.br

\section{Luciana R. Hirsch}

Departamento de Física, Universidade Tecnológica Federal do Paraná Av. Sete de Setembro, 3165, 80230-901 Curitiba-PR, Brasil

E-mail: lhirscheutfpr.edu.br

\section{Antonio Delfino Jr}

Instituto de Física, Universidade Federal Fluminense

Av. Gal. Milton Tavares de Souza, 24210-346 Niterói-RJ, Brasil

E-mail: delfinodif.uff.br

\begin{abstract}
A thermal hadronic model including two different temperatures to describe the freeze out of the expanding fireball in a ultrarelativistic heavy ion collision is presented. We introduced distinct effective freeze out temperatures for the baryonic and mesonic sectors of expanding fireball. The model is applied to determine and to fit particle population ratios of hadrons produced in the reaction. The proposal is not merely incorporate one additional degree of freedom to the adjustment procedure of data within thermal model, but to stress out a different scenario for the freeze out stage in the collisional process. The reformulated version of thermal model was applied to a set of consolidated data in the literature, offering a rather good improvement in the fitting quality of calculated particle ratios to the data.
\end{abstract}

XXXIV edition of the Brazilian Workshop on Nuclear Physics,

5-10 June 2011

Foz de Iguaçu, Paraná state, Brasil

\footnotetext{
${ }^{*}$ Speaker.

${ }^{\dagger}$ A footnote may follow.
} 


\section{INTRODUCTION}

A significant amount of experimental data for particle production in high energy heavy ion collisions (10 - $200 \mathrm{GeV} / \mathrm{A}$ at center of mass) has been accumulated. Many different theoretical attempts have tried to described these data using thermal models in the approximation of global thermal equilibrium considering only one freeze-out temperature [1]-[14]. Because of its simplicity, the model is well acceptable to treat and explain the data. However the thermal models often are not able to describe adequately all multiplicities of hadrons [4], for instance, the abundance of strange particles are overestimate and the pion yields are underestimated. In addition, particle number density and total energy density calculated in chemical freezing are overestimated [9]. To overcome these limitations some features have been incorporated in the model, as for example the inclusion of a phenomenological parameter $\gamma_{s}$ to adjust the strangeness production in the reaction process [5]-[7], or the inclusion of effects of excluded volume [8], [9] , [10]. However, the main criticism is that the oversimplified conception of the model makes the connection of the model with more realistic view of the dynamical evolution a hard task. For example, the hydro model [15] or non equilibrium kinetic equation treatments conceptually do not mash with conventional thermal model image. More recent lattice results has been shown that hadronization critical temperature differ significantly from chemical freeze out temperature estimated by thermal model by tens of $\mathrm{MeV}$ [16]-[18].

The question of whether hadrons are born out of chemical equilibrium has been arisen previously to justify the difference between critical and freeze out temperatures before the RHIC results. However, the used arguments were not strong enough to be compatible with RHIC results few years latter [19]-[20]. The solution is still no conclusive but some effort have been made more recently [21] trying to justify the indicative of a small relaxation time of the hadron chemical equilibrium after their born at critical temperature at RHIC. The main idea is to recall the Hagedorn's states, introduced in the 1960's, to quickly drive hadrons to chemical equilibrium [19]. The introduced intermediate resonant state (included in the game) should have a mass spectrum defined by the Hagedorn's temperature, assumed to be adjustable parameter in the statistical calculation.

In present work we decidedly assume that the freeze-out temperature of the thermal model should be considered as being an effective thermodynamic quantity associated to the expanding stage of the system evolution. Indeed, there is no confident estimative to the temperature changes in expanding system starting from the occurrence of hadronization process till to the freeze out phase. Also we have no well-founded reason to support the statement of equal thermal evolution of mesons and baryons sectors between these stages. Consequently, only simplicity arguments basis the conventional thermal model assumption of a common thermal environment for these sectors at the freeze out. A more reasonable working hypothesis would be different effective dynamical freeze out temperatures for these distinct sectors. One argument in favor of this scenario is that after hadronization the chemical equilibrium (if established) is maintained (or reached) dominantly by mean of the meson-baryon and baryon-baryon processes, having differentiated values of cross sections (with baryon-baryon relaxation favored with a large cross section).

The idea of use explicitly two distinct temperature in the thermal model is not new and has been revisited more recently in the literature [22]-[24]. Here we are attributing these effective temperatures to the different hadronic sectors of the system. In addition, the hypothesis of two 
temperatures can also be thought as a consequence of a temperature distribution in the fireball at the freeze out. Note that more realistic than have an isothermal fireball is to admit that the fireball presents an inner region dominated by baryons (with higher effective temperature) and an outer layer, with lower effective temperature dominated by mesons, an image more compatible with hydro or transport calculations [25]-[30].

The two effective temperatures evoked for the thermal model still preserves the relatively simple model skill to perform calculation, bringing the this kind of approach closer to the more detailed description of the fireball expansion. Having in mind this qualitative arguments, our purpose in this work is to study particle population ratios using thermal model with this alternative freeze-out scenario. A first condition required to this alternative model is an improving of quality of results obtained with conventional calculation. Thus as a preliminary task we have apply the two-temperature model to different collisional processes with consolidated data set of particle ratios in the literature. We have also analyse the quality of the results in comparison with those ones obtained with the conventional model.

In the next section we will make a detailed presentation of two-temperature thermal model applied to the determination of particles population. In Section 3 we show predictions for particle produced ratio from the modified model compared with the results of conventional thermal calculation. Finally, in the last section we discuss the improvement in the quality of data fitting provided by the proposed model.

\section{HADRONIC THERMAL MODEL WITH TWO TEMPERATURE}

In thermal models the particle density of the $k$-th particle is obtained taking into account the distribution functions of baryons and meson given by the Fermi-Dirac and Bose-Einstein distributions functions respectively,

$$
n_{k}=\frac{\gamma_{k}}{2 \pi^{2}} \int \frac{p^{2}}{\exp \left[\left(\varepsilon_{k}-\mu_{k}\right) / T_{i}\right] \pm 1} d p
$$

where $\varepsilon_{k}=\sqrt{p^{2}+m_{k}^{2}}$, the minus (plus) sign stands for the Fermi-Dirac (Bose-Einstein) distribution, $\gamma_{k}$ is the spin degeneracy and $\mu_{k}$ is the chemical potential. $T_{i}(i=b, m)$ is the baryonic $(i=b)$ or mesonic $(i=m)$ temperature to be replaced in Eq. (2.1) according to the type of particle. The chemical potential of the $k$ th particle $\mu_{k}$ is calculated using the global chemical potential $\left\{\mu_{B}, \mu_{I 3}, \mu_{S}\right\}$ as $\mu_{k}=B_{k} \mu_{B}+I 3_{k} \mu_{I 3}+S_{k} \mu_{S}$, where $\left\{B_{k}, I 3_{k}, S_{k}\right\}$ are the baryonic, isospin and strageness numbers of the particle. The global chemical potentials are constrained to the conservation of baryonic, isospin and strangeness charges as

$$
\begin{aligned}
V \sum_{k} B_{k} n_{k} & =Z_{1}+Z_{2}+N_{1}+N_{2} \\
V \sum_{k} I 3_{k} n_{k} & =\frac{1}{2}\left(Z_{1}+Z_{2}-N_{1}-N_{2}\right) \\
\sum_{k} S_{k} n_{k} & =0
\end{aligned}
$$


where $Z_{i}$ and $N_{i}(i=1,2)$ are the number of protons and neutrons of the colliding nuclei and $V$ is the volume of the fireball.

The complete baryonic decuplet and mesonic nonet are included, together with around two hundred of other baryonic and mesonic resonances with masses up to $2 \mathrm{GeV}$. It is also important to point out that final particle population calculated by Eqs. (2.1-2.4) (to be compared to data) should be changed due to the decay chain of included baryon and meson resonances, a process known as the feed-down of particles population. We reformulated the resonance decay feeding processes of the conventional thermal model to make it consistent with the two distinct freeze out temperatures. We have used the well known equations,

$$
n_{k}=n_{k}^{(t)}+\sum_{j} \Gamma_{j \rightarrow k} n_{j}^{(t)},
$$

where $n_{k}^{(t)}$ is the thermal particle density calculated using Eq. (2.1), and $\Gamma_{j \rightarrow k}$ is the probability of particle $j$ to decay into particle $k$, from experimental data. Note that each thermal population is calculated using a specific effective temperature in Eq. (2.1). Thus, the the decay processes equations are affected by the use of two different temperatures for the hadronic sectors. Only the decuplet and nonet resonances are considered to contribute significantly to the feed-down by their strongly decay channels.

\section{RESULTS AND DISCUSS}

To compare results obtained from conventional model with those from two-temperature present here, we have performed the conventional calculation by using the elaborated two-temperature code under the constraint, $T_{b}=T_{m}$. The two-temperature code was constructed from a previous version of thermal model developed by some of the present authors [13], [14]. Results are shown in Table I and II for comparison, using three systems in collision and corresponding data of particles population ratio. The first system data (set C1) is taken from Ref. [3], as well the second set (set C2). The third data set (C3) was extracted from Ref. [11]. In Table 1 we present the parameters values for the best data fitted through the conventional thermal model.

\begin{tabular}{|c|c|c|c|c|c|}
\hline Experimental Sets & $T(\mathrm{MeV})$ & $\mu_{b}(\mathrm{MeV})$ & $\chi_{\text {d.o.f }}^{2}$ & $\sqrt{s}(\mathrm{GeV})$ & Ions \\
\hline$C 1$ & $152.6_{(11.3)}^{(11.2)}$ & $271.9_{(26.0)}^{(24.5)}$ & 4.494 & $17.3 A$ & $S i+S i$ \\
\hline$C 2$ & $147.4_{(2.6)}^{(4.35)}$ & $287.2_{(16.4)}^{(14.9)}$ & 1.667 & $17.3 A$ & $P b+P b$ \\
\hline$C 3$ & $29.6_{(0.05)}^{(0.63)}$ & $772.9_{(0.25)}^{(7.25)}$ & 0.160 & $2.32 A$ & $N i+N i$ \\
\hline
\end{tabular}

Table 1: Results for the thermodynamic parameters for different collisions using conventional thermal model. The error bars in the temperature and baryonic chemical potential are established as the isolated variation in these quantities which leads to a $10 \%$ of change in the optimal $\chi_{\text {d.o.f }}^{2}$ value.

In table II we present the model parameter values for the best fitting of calculated ratio of particles to the set of data in table I, now using the two-temperature approach for the freeze out.

From the comparison of the above tables we see that in all analysed systems both effective temperature in Table II $\left(T_{b}\right.$ and $\left.T_{m}\right)$ are lower than the single freeze out temperature in table I (T). 


\begin{tabular}{|c|c|c|c|c|c|c|}
\hline Experimental Sets & $T_{m}(\mathrm{MeV})$ & $T_{b}(\mathrm{MeV})$ & $\mu_{b}(\mathrm{MeV})$ & $\chi_{\text {d.o.f }}^{2}$ & $\sqrt{s}(\mathrm{GeV})$ & Ions \\
\hline$C 1$ & $115.0_{(2.40)}^{(0.60)}$ & $127.8_{(0.4)}^{(2.0)}$ & $190.56_{(27.16)}^{(3.34)}$ & 0.9999 & $17.3 A$ & $S i+S i$ \\
\hline$C 2$ & $133.36_{(0.44)}^{(1.96)}$ & $140.05_{(1.85)}^{(0.20)}$ & $251.80_{(12.80)}^{(6.60)}$ & 0.9997 & $17.3 A$ & $P b+P b$ \\
\hline$C 3$ & $27.9_{(0.09)}^{(2.19)}$ & $29.6_{(0.48)}^{(0.24)}$ & $772.0_{(10.35)}^{(1.0)}$ & 0.9666 & $2.32 A$ & $N i+N i$ \\
\hline
\end{tabular}

Table 2: Thermodynamic parameters for different collisions obtained by using the two-temperatures model. The error bars are defined as previously in table I.

Another direct observation is that the fits in table II, reflected by the $\chi_{\text {d.o.f }}^{2}$ values, presents a rather good quality compared with those in Table I.

In Figure 1 we show three plots for the system $\mathrm{Pb}+\mathrm{Pb}$ at $17.3 \mathrm{GeV}$.A, where it is represented results for the adjusted model parameters with $0.95<\chi_{\text {d.o.f }}^{2}<1.1$. Each generated point corresponds to values of $T_{b}, T_{m}$ and $\mu_{b}$ in the multiparametric space. In part (a) the difference $\delta T=T_{b}-T_{m}$ are plotted against the baryonic chemical potential. A set of five hundred points are generated covering the domain of points with acceptable $\chi_{\text {d.o.f }}^{2}$ values. In part (b) we depicted the same difference against the baryonic effective temperature, and in c) we show the points in the plane $\left(T_{b} ; T_{m}\right)$. We call attention to the part (a) and (b) of the plots, where the elliptical area generated cover a wide range of $\delta T$, but does not reach $\delta T=0$. This means that the model with only one temperature is not able to reproduce data with the specified optimal quality level of the fitting. Same situation was observed from similar plots (not shown) for the others analysed systems.

In figures 2 and 3 we show the obtained particles ratios result for the best fitting compared with the experimental data for the analysed system in collision. We can see the excellent agreement between our predictions and experimental data.
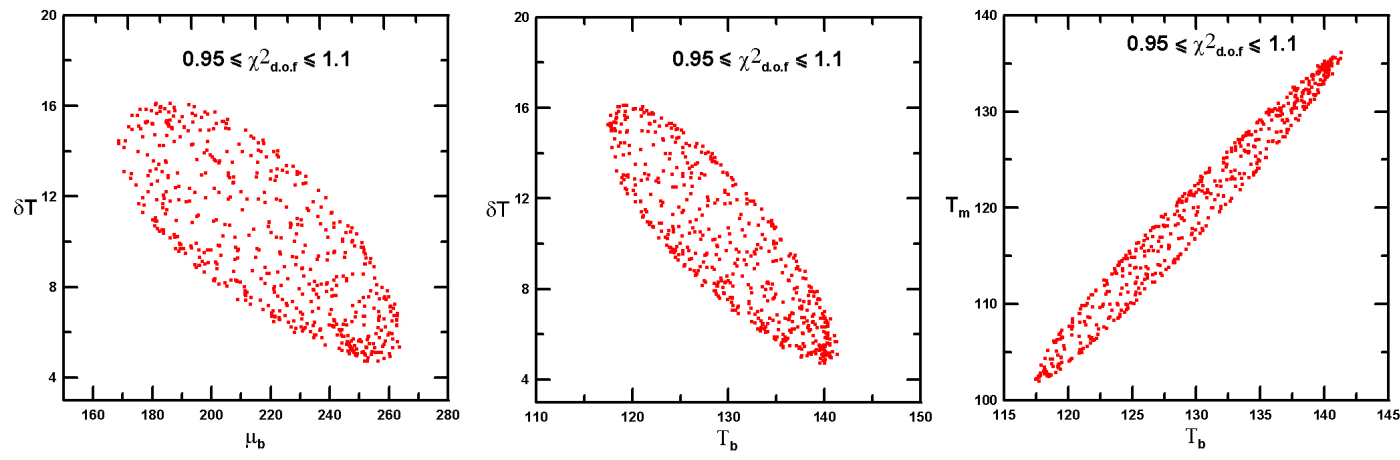

Figure 1: Plots for thermodynamic quantities in events of $\mathrm{Pb}+\mathrm{Pb}$ collisions with quality of the fits given by $\chi_{\text {d.o.f }}^{2}$ between 0.95 and 1.1 . In part (a) we have $\delta T$ against the baryonic chemical potential. In part (b) we have $\delta T$ against the baryonic effective temperature and in part (c) we show the points in the $\left(T_{b} ; T_{m}\right)$ parameter plane. 

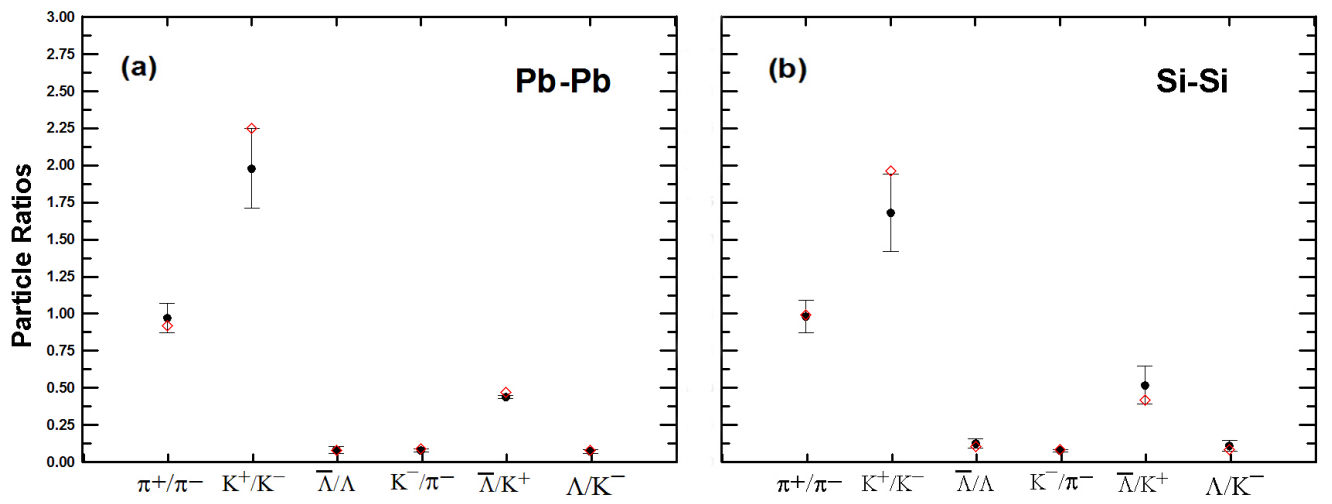

Figure 2: Plots for particle ratios in events of $\mathrm{Pb}+\mathrm{Pb}$ (a) and $\mathrm{Si}+\mathrm{Si}$ (b) collisions at $\sqrt{s}=17.3 \mathrm{~A} \mathrm{GeV}$. The black circles with error bars are the experimental values [3] and red diamonds are the theoretical predictions from two temperatures model.

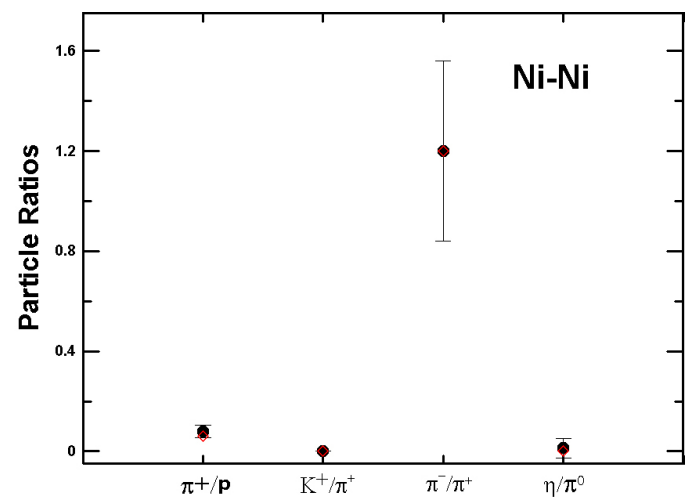

Figure 3: Plots for particle ratios in events of $\mathrm{Ni}+\mathrm{Ni}$ collisions at $\sqrt{s}=2.32 \mathrm{~A} \mathrm{GeV}$. The black circles with error bars are the experimental values [11] and red diamonds are the theoretical predictions from two temperatures model. 


\section{CONCLUSION}

The above results seem very promising and encouraging for explore a more comprehensive data sets existing in the literature. A selection of more consolidated data are in progress since it is usual to have in the literature different data presentations coming from the same experiment, but submitted to differentiated filter or criteria in debugging data. In general for a given reaction the data set of the particle population rate includes only few different particle ratios and it is a hard task to obtain confident model fitting to it.

In our calculation (see results in figures 1-3) we have shown that with the two-temperature thermal model we can obtain excellent fits for the ratios of particles population in a quite wide region of acceptable parameters values. This aspect open a room to accommodate in the model predictions for other reaction observable such as strangeness production, transverse distribution of momentum, total particle-antiparticles ratios, and others. This suggest that the implemented improvement in the model makes the thermal approach more robust to handle with a larger number of colliding system and more comprehensive set of reaction observables. Presently we are working toward this direction in order to certify the proposed model.

\section{References}

[1] I. Kraus, J. Cleymans, H. Oeschler, K. Redlich, S. Wheaton, Statistical model predictions for collisions at LHC, Progress in Particle and Nuclear Physics 62, 2, 538-542, 2009.

[2] I. Kraus, J. Cleymans, H. Oeschler, K. Redlich, Particle production in p p collisions and predictions for $\sqrt{s}=14$ TeV at the Cern Large Hadron Collider (LHC), Physical Review C 79, 1, 014901, 2009.

[3] I. Kraus, J. Cleymans, H. Oeschler, K. Redlich and S. Wheaton, Chemical equilibrium in collisions of small systems, Physical Review C 76, 014901, 2009.

[4] J. Cleymans, K. Redlich, H. Satz and E. Suhonen, The hadronization of a quark-gluon plasma, Zeitschrift fur Physik C Particles and Fields, 58, 2, 347-355, 1994.

[5] J. Rafelski, Strange anti-baryons from quark-gluon plasma, Physics Letters B, 262, 2-3, 333-340, 1991.

[6] F. Becattini, A thermodynamical approach to hadron production in $e^{+} e^{-}$collisions, Zeitschrift fur Physik C Particles and Fields, 69, 3, 485-492, 1996.

[7] F. Becattini, M. Gazdzicki and J. Sollfrank, On chemical equilibrium in nuclear collisions, The European Physical Journal C - Particle and Fields, 5, 1, 143-153,1998.

[8] D. H. Rischke, M. I. Gorenstein, H. Stöcker and W. Greiner, Excluded volume effect for the nuclear matter equation of state, Zeitschrift fur Physik C Particles and Fields, 51, 3, 485-489, 1991.

[9] G. D. Yen and M. I. Gorenstein, Analysis of particle multiplicities in Pb+Pb collisions at 158A GeV/c within hadron gas models, Physical Review C, 59, 2788, 1999.

[10] P. Braun-Munzinger, I. Heppe, J. Stachel, Chemical equilibration in $\mathrm{Pb}+\mathrm{Pb}$ collisions at the SPS, Physics Letters B, 465, 15, 1999.

[11] J. Cleymans, H. Oeschler, and K. Redlich, Influence of impact parameter on thermal description of relativistic heavy ion collisions at $(1-2 A)$ GeV, Physical Review C 59, 3, 1663, 1999. 
[12] A. Andronic, P. Braun-Munzinger, J. Stachel, Thermal hadron production in relativistic nuclear collisions: The hadron mass spectrum, the horn, and the QCD phase transition, Physics Letters B, 673, 2, 142-145, 2009.

[13] L. R. Hirsch, Modelo Térmico em Colisões Nucleares em Altas Energias, PhD - Thesis - UFF-Brazil, 2010.

[14] L. R. Hirsch, A. Delfino, M. Chiapparini, Hadron Production in a Thermal Model with Scaled Masses, Nuclear Physics B-Proceedings Supplements, 199, 297, 2010.

[15] A. Muronga, Second-order dissipative fluid dynamics for ultrarelativistic nuclear collisions, Physical Review Letters, 88, 6, 062302, 2002.

[16] R. Rapp and E. V. Shuryak, Resolving the Antibaryon-Production Puzzle in High-Energy Heavy-Ion Collisions, Physical Review Letters, 86, 2980, 2001.

[17] C. Greiner, Importance of multimesonic fusion processes on (strange) anti-baryon production, AIP Conference Proceedings, 644, 337, 2003; Do chemically saturated anti-hyperon abundancies signal the quark gluon plasma?, Heavy Ion Physics, 14, 149 2001; C. Greiner and S. Leupold, Antihyperon-Production in Relativistic Heavy Ion Collision, Journal of Physics G, 27, L95, 2001.

[18] P. Koch, B. Muller, and J. Rafelski, Strangeness in relativistic heavy ion collisions, Physics Reports, 142, 1671986.

[19] J. I. Kapusta and I. Shovkovy, Thermal rates for baryon and anti-baryon production, Physical Review C, 68, 014901, 2003; J. I. Kapusta, Thermal rates for baryon and anti-baryon production, Journal of Physics G, 30, S351, 2004.

[20] P. Huovinen and J. I. Kapusta, Rate equation network for baryon production in high energy nuclear collisions, Physical Review C, 69, 014902, 2004.

[21] J. Noronha-Hostler, H. Ahmad, J. Noronha, Physical Review C, Particle ratios as a probe of the QCD critical temperature, 82, 024913, 2010.

[22] Z. D. Lu, B. H. Sa, A. Faessler, C. Fuchs, E. E. Zabrodin, Analysis of multi-particle production at RHIC by two-source statistical model, High Energy Physics and Nuclear Physics-Chinese, 26, 11, 1166, 2002.

[23] Z. D. Lu, B. H. Sa, A. Faessler, C. Fuchs, E. E. Zabrodin, Analysis of particle production in ultrarelativistic heavy-ion collisions within a two-source statistical model, Physical Review C, 66, 4, 044905, 2002.

[24] S. Choi, K. S. Lee, Two freeze-out model for hadrons produced in the relativistic heavy-ion collisions, Physical Review C, 84, 064905, 2011, Physical Review C, 84, 069901,2011 and [ArXiv: 1107.2484v1].

[25] S. A. Bass and A. Dumitru, Dynamics of hot bulk QCD matter: From the quark-gluon plasma to hadronic freeze-out, Physical Review C, 61, 064909, 2000.

[26] H. Song, S. A. Bass and U. Heinz, Viscous QCD matter in a hybrid hydrodynamic Boltzmann approach, Physical Review C, 83, 024912, 2011.

[27] T. Hirano and K. Tsuda, Collective flow and two-pion correlations from a relativistic hydrodynamic model with early chemical freeze-out, Physical Review C, 66, 054905, 2002.

[28] H. Bebie, P. Gerber, J.L. Goity, H. Leutwyler, The role of the entropy in an expanding hadronic gas, Nuclear Physics B, 378, 1992. 
[29] P. F. Kolb, R. Rapp, Transverse flow and hadrochemistry in Au+Au collisions at $\sqrt{s_{N N}}=200 \mathrm{GeV}$, Physical Review C, 67, 044903, 2003.

[30] P. Huovinen, Chemical freeze-out temperature in the hydrodynamical description of Au+Au collisions at root $\sqrt{s_{N N}}=200 \mathrm{GeV}$, European Physics Journal A, 37, 121, 2008. 